\title{
Research and Implementation of Multi-system Voice Convergence
}

\author{
Linying Geng, Lingle Huo, Hongwei Qin, Weiwei QI, Ming Guo, Shutao Dong \\ China Luoyang Experiment Center of Electronic Equipment, 471023, China
}

Keywords: commanding communication, voice service, convergence processing, technical improvement, design

\begin{abstract}
. since there is a problem that commanding communication have different kinds of terminals and the existing system to guarantee voice commanding communication cannot be "one-size-fits-all”, this paper, under the condition that the dispatch terminal is as the relay and the current dispatching system is as the switching system, describes the technical improvement and design to a simple convergence device in terms of its interface and signaling transfer. By controlling and transferring the audios, convergence system can overcome the barriers resulted in by the technical protocols owned by different sound systems, so that the intercommunication and networking application among different sound systems can be available; what's more, guarantee mode of speech communication is simplified and the command efficiency is enhanced. Therefore, the existing communication equipment can be better made use of. After being verified and accepted, it is proved that technical improvement described in this paper can take good effect.
\end{abstract}

\section{Introduction}

In the information era, it is mandatory for us to think about several factors like regions, environment, security and confidentiality etc. In order to make sure that the orders from the commander can be correctly delivered, a multi-system commanding communication system is required to be set up. Such a system will make the measures to guarantee the communication diversified and the ability in guaranteeing communication strengthened. However, different sound systems use different technologies and these sound systems are independent from each other. That is these systems do not have intercommunication and resource integration is not realized[1]. If all sound systems can have intercommunication with each other, they can be considered to be a whole. If so, the commanding terminal of the commander is able to give orders to all operation terminals. In this way, an effect "one-size-fits-all" can come true and the commanding efficiency will be maximally enhanced. Under this background, it is badly needed to study the multi-system convergence for commanding communication, try to integrate all the existing communication systems. Anyway, it is mandatory to get all systems can have intercommunication with each other and enhance the commanding efficiency.

\section{Problems encountered by existing modes to guarantee voice commanding communication}

For current communication guarantee, there are several voice communication systems, which cannot have intercommunication with each other. Normally, these systems will encounter 2 major problems:

First, ability in commanding and dispatching is not sufficient enough. Today, most commanding dispatch systems are supported by cables, which mainly covers the dispatching terminal equipped in the commanding building. That is the existing commanding dispatch systems are always restricted by methods of transmission and quantity of terminals. Because of such restrictions, the ability in commanding and dispatching is quite limited. Once the points to be commanded are decentralized and tasks are diversified, the current commanding dispatch system cannot meet the actual needs.

Second, the current commanding dispatch systems are not convenient to operate. When commanding and dispatching, the commander shall have several terminals available, like fixed-line telephone system, trunk system handset and dispatch terminal, etc. Therefore, the commander shall well remember the pairing of these terminals. Sometimes, the commander needs to repeat the same 
order for several times in order to deliver the order to different people. As a result, the operation is not convenient and the work efficiency is low. What's worse, it is easy to have errors[2].

In order to overcome the problems mentioned above, it is badly required to realize the intercommunication among different voice systems (commanding dispatch system, fixed-line telephone and trunk system), trying to have an effect of “one-size-fits-all”.

\section{Technical improvement of multi-system convergence system for commanding communication voice service}

\subsection{Introduction of audio convergence device}

The research of this paper is based on BY-AVSP-TS004-1 audio fusion dispatching device (hereinafter referred to as "audio fusion device"). The audio fusion device is mainly composed of universal controller chassis and various boards. The universal controller chassis is the container of various boards. It supplies power for boards, provides data exchange among boards, and provides the corresponding external interface for each board. However, the audio fusion device only equips with two function of that program-controlled telephone dials four-wire analog dispatching, and program-controlled telephone calls cluster handset. And it doesn't equip with the function of three party service. It cannot achieve those functions of dispatch terminal, cluster base station, interconnection of Program - controlled exchange and hybrid audio conference.

\subsection{Design ideology}

The existing equipment cannot provide three-party conference because: the cable commanding dispatch system is a kind of digital dispatching system, which only supports $\mathrm{H} .323$ protocol ${ }^{[2]}$, the convergence device uses SIP protocol. Therefore, the cable commanding dispatch system cannot intercommunicate with the convergence device. The wireless trunk system being used has several protocols, so the protocols set by convergence device supplier and trunked dispatch system supplier might be different from each other. If intercommunication among the systems needs to be realized, it is mandatory to overcome the protection done to the private protocols set by different suppliers. That is it is necessary to the audios shall be controlled and transferred after being extracted.

Under this background, here forms the design ideology [3] of multi-system convergence system for different voice services: under the condition that digital dispatch system is the core switching system, audio convergence device is the foundation, make sure that the existing voice commanding communication system is being normally operated, try to do the technical improvement on some equipment interfaces. Make a dispatch terminal as the relay to get the trunk system and programmed control system shall intercommunicate with each other. Trunk system and fixed-line telephone shall intercommunicate with a dispatch terminal respectively, then the dispatch terminal will collect the audio signal from each equipment. Meanwhile, the dispatch terminal will connect with host dispatch switch via its own internet access, by which the intercommunication among trunk system, programmed control telephone system and commanding dispatch system will be realized. In this way, "one-size-fits-all” can be available, which will enlarge the guarantee ability of all voice systems and enhance the commanding efficiency, as well as enhance the efficiency of original devices. Fig. 1 shows how a cable commanding dispatch terminal communicates with a set of communication system. 


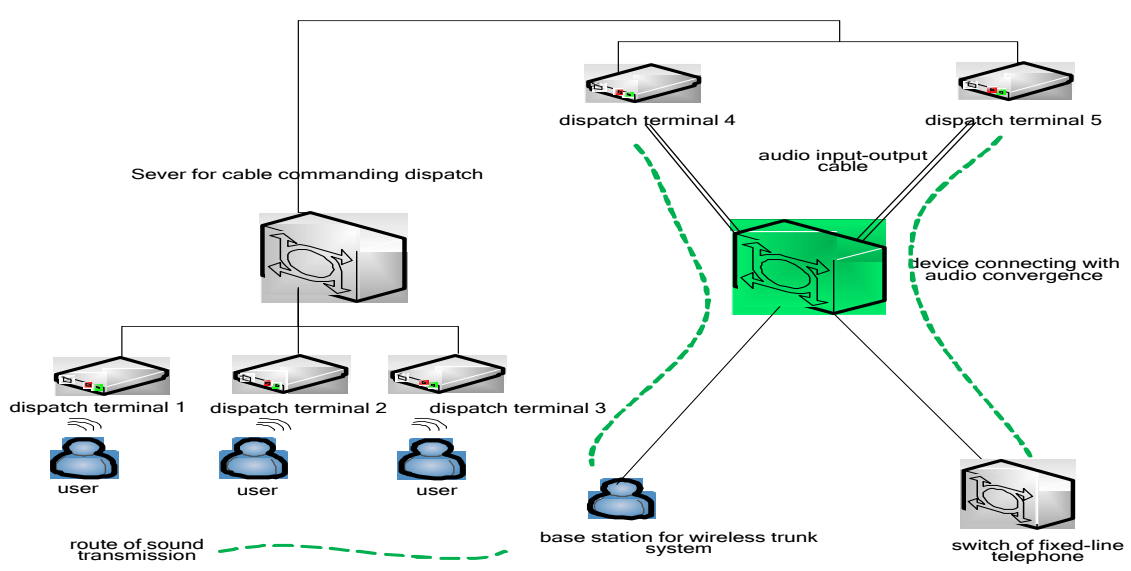

Fig. 1 One Cable Commanding Dispatch Terminal Communicates with a Set of Communication System

\subsection{Interface design}

For base station of trunk system, internet access, serial port, loop trunk, E1 access, user interface, audio input interface and PTT input interface are all available. In this paper, the base station of trunk system is connected with convergence device via loop interface. First, software fix shall be done to relevant interfaces of convergence device. How will convergence device use software? Step 1, dial the internal number of convergence device; step 2, dial the number of telephone network; step 3, dial the dispatching center of trunk system; step 4, dial handset or vehicle-locating set. Then the interconnection among these systems can be realized. Fig. 2 is the connection between trunk system and audio convergence device.

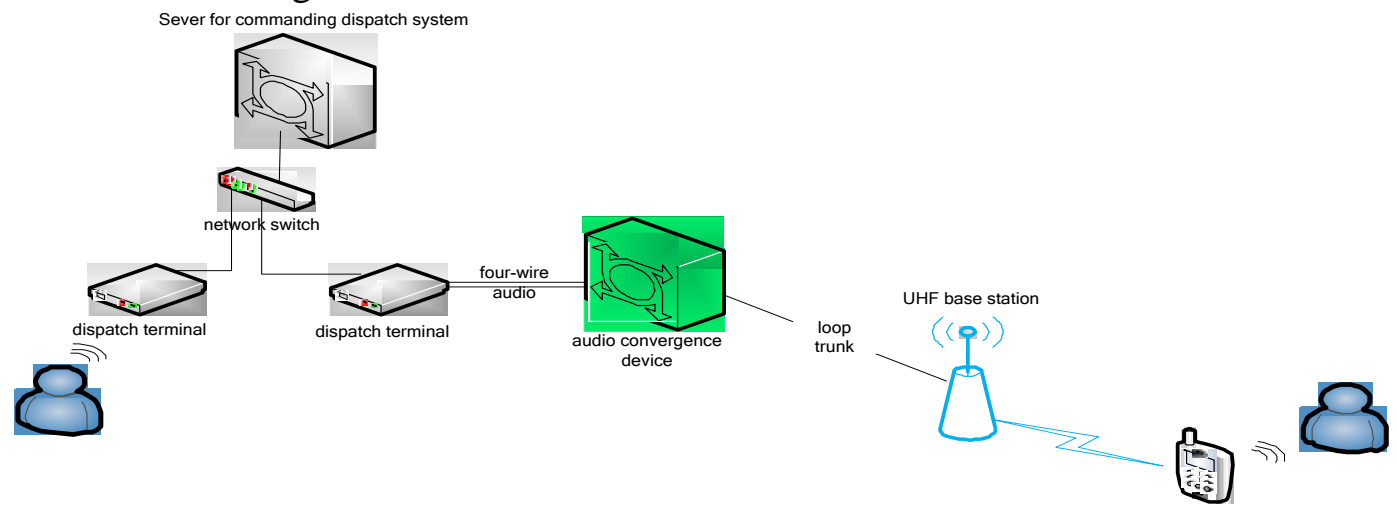

Fig. 2 Connection between Trunk System and Audio Convergence Device

The convergence device is able to connect a special trunk system, which does not support independently-used trunk system (hereafter referred to as “UHF”). Since UHF provides a loop trunk, once there are telephone lines, an intercommunication with UFH can be realized. In order to easily access to UHF, fixed-telephone lines given to the users will be applied to access to UHF. At the same time, audio convergence device will be accessed to fixed-line telephone network via E1 loop trunk. Thus, intercommunication between audio convergence device and UHF. Fig. 3 is the intercommunication between UHF and commanding dispatch system via fixed-line telephone network. 


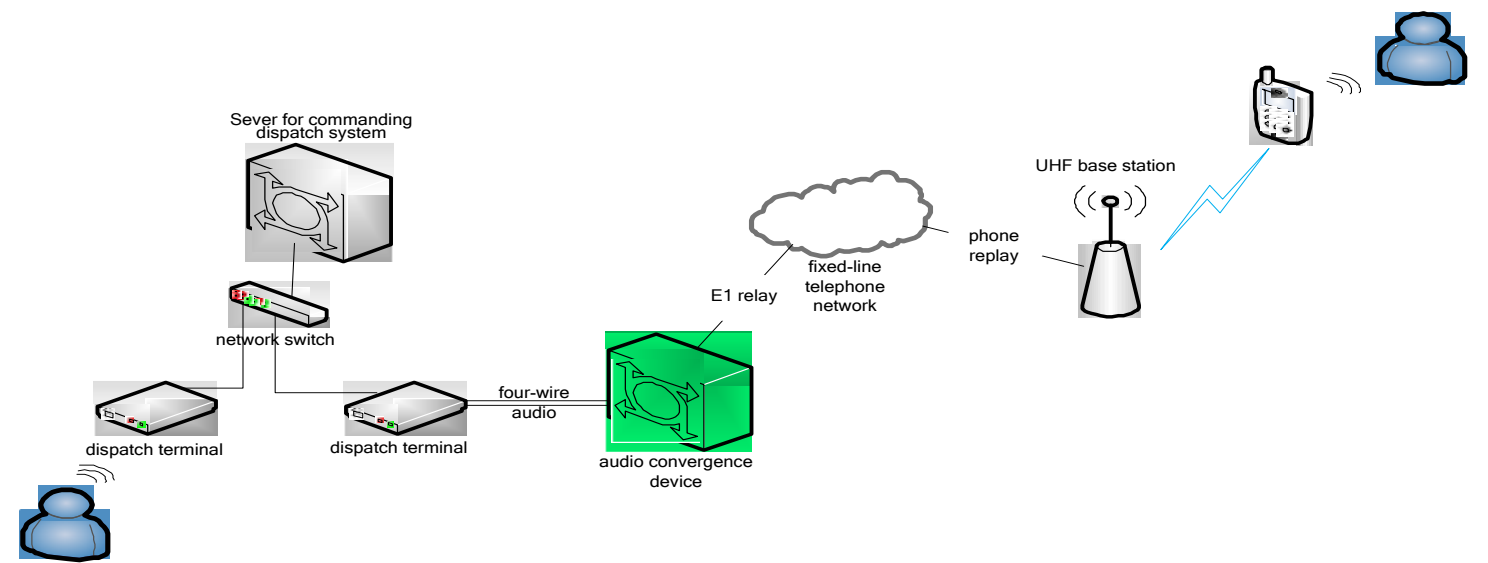

Fig. 3 Intercommunication between UHF and Commanding Dispatch System [4]

However, accessing to wireless group via loop trunk is still being restricted by time. This restriction is resulted in by the features of the equipment. In order to access to UHF, much time is spent studying the UHF equipment. According to the study, UHF is to be accessed to fixed-line telephone network, which well avoid the defects owned by UHF equipment. Therefore, the restriction by time is solved and stable access to a single wireless user is realized.

\section{Implementation of improved multi-system convergence for commanding communication} voice service

\subsection{Signaling transfer}

Audio convergence system can mainly realize the intercommunication among commanding dispatch system, trunk system and fixed-line telephone system [5]. For commanding dispatch system, a dispatch terminal is used as the relay for audio intercommunication. For audio convergence device, the intercommunication is realized by connecting the audio wire with audio input-output terminals on the dispatch terminal. Trunk system will intercommunicate with audio convergence system via loop trunk. Fixed-line telephone will intercommunicate with audio convergence device via E1 interface.

Signaling transfer of audio convergence system is realized by [6] ISG of audio convergence device. ISG consists of several elements, including GCU, DTU, ATU, ASU, ECU, MRU and CMU etc.

DTU can get the digital trunk accessed (including the access of signaling 7), by which the intercommunication between audio convergence system and fixed-line telephone network via DTU. DTU is located at MGU. Refer to Fig. 4 for the signaling from fixed-line telephone network.

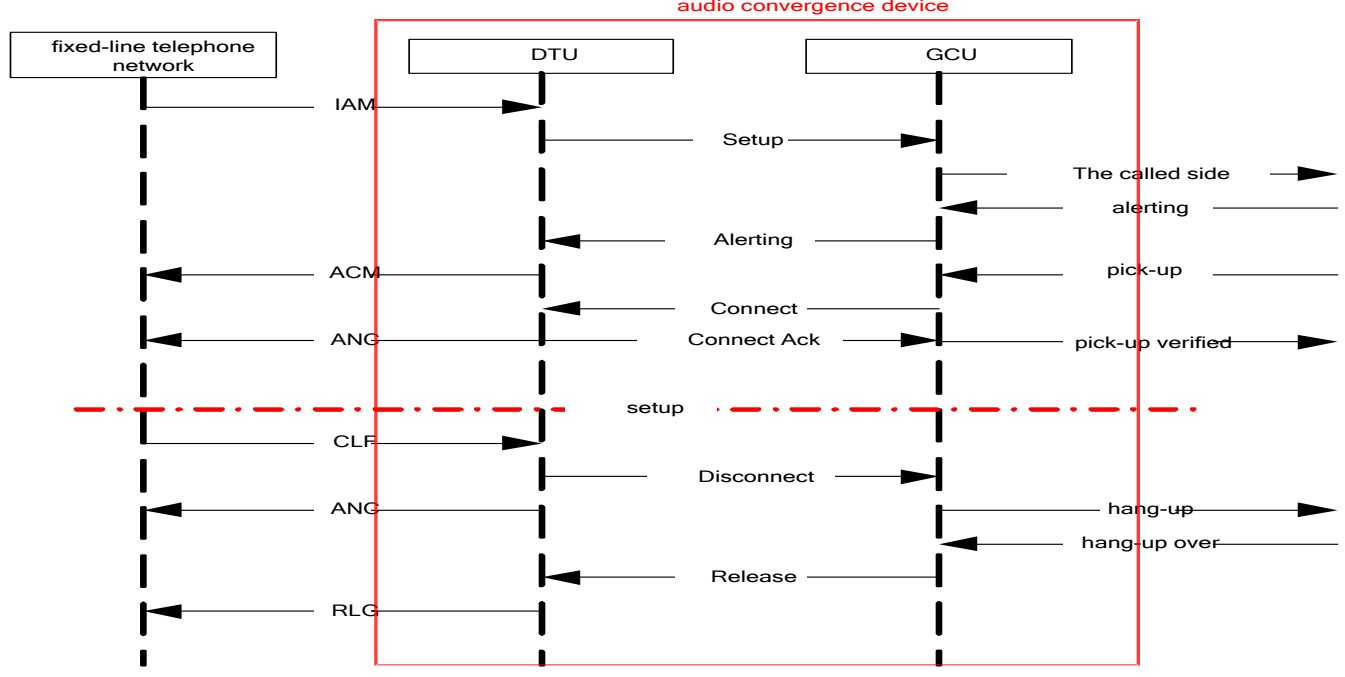

Fig. 4 Call-in of Fixed-line telephone network 
ATU can get the loop trunk accessed. For audio convergence system, an intercommunication with trunk system will be realized via ATU. Then by such an intercommunication, some functions can be available --- detect events, that is ATU will get the events adapted to the standard procedures on GCU and fulfill the tasks by the control GCU. ATU is located at DLC. Refer to Fig. 5 for the signaling from loop trunk call-in.

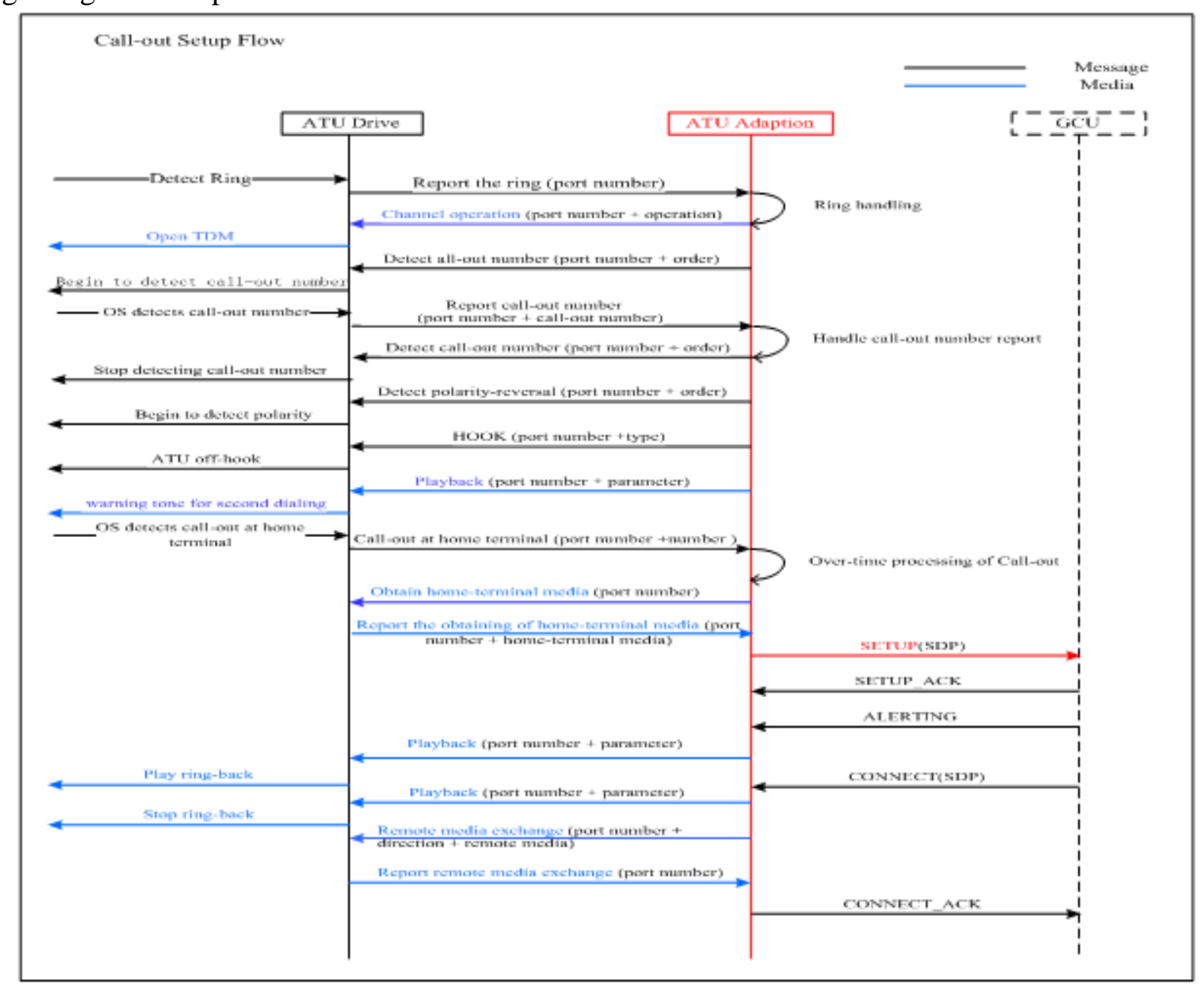

Fig. 5 Loop Trunk Call-in Flow

ECU can get wireless equipment (including wireless host security dispatch terminal, encryption equipment, adaptive controller, receiver, sender etc) accessed. By ECU, intercommunication among commanding dispatch system and wireless dispatch trunk system can be realized. ECU is mainly used to detect the events occurred at audio interface, PTT and RS232 etc. Then, ECU will get the events adapted to the standard control procedures on GCU and fulfill the tasks by the control of GCU. ECU is located at MT-ECU4321. Refer to Fig. 6 for call-out from audio device via ECU. 
Audio convergence device

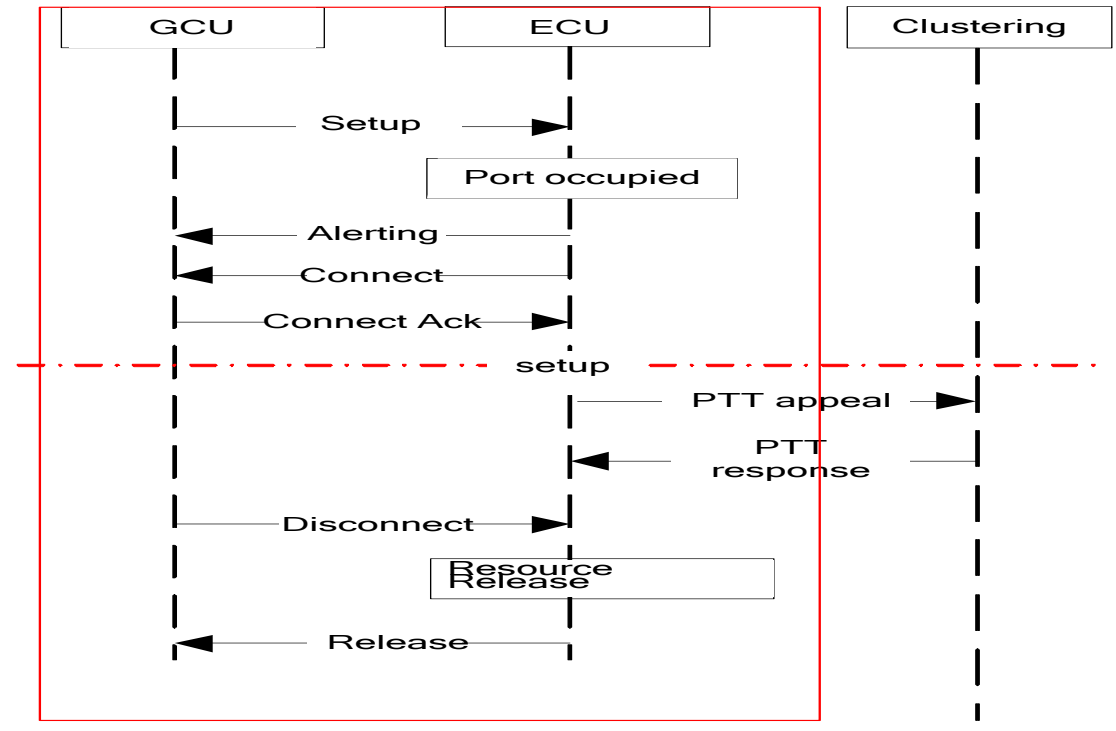

Fig. 6 Call-out clustering Procedure of Audio Convergence Device

\subsection{Media Exchange Procedure}

If simulated voice signal needs to be transmitted via IP network, it is mandatory to have analogue / digital exchange first; after the analogue/digital exchange, the coded data shall be compressed into a RTP package; then the RTP package will be transmitted to the targeted terminal via IP network [7]. After receiving the compacted data, the targeted terminal will decompress RTP packages; then, the decompressed data will be transferred after digital / analogue exchange.

As for digital audio signals to be transmitted via E1 and other routes, they will be transmitted to the audio output port after time slot interchange. Then, the signals will have digital / analogue exchange done at the targeted terminal.

Refer to Fig. 7 for media exchange.

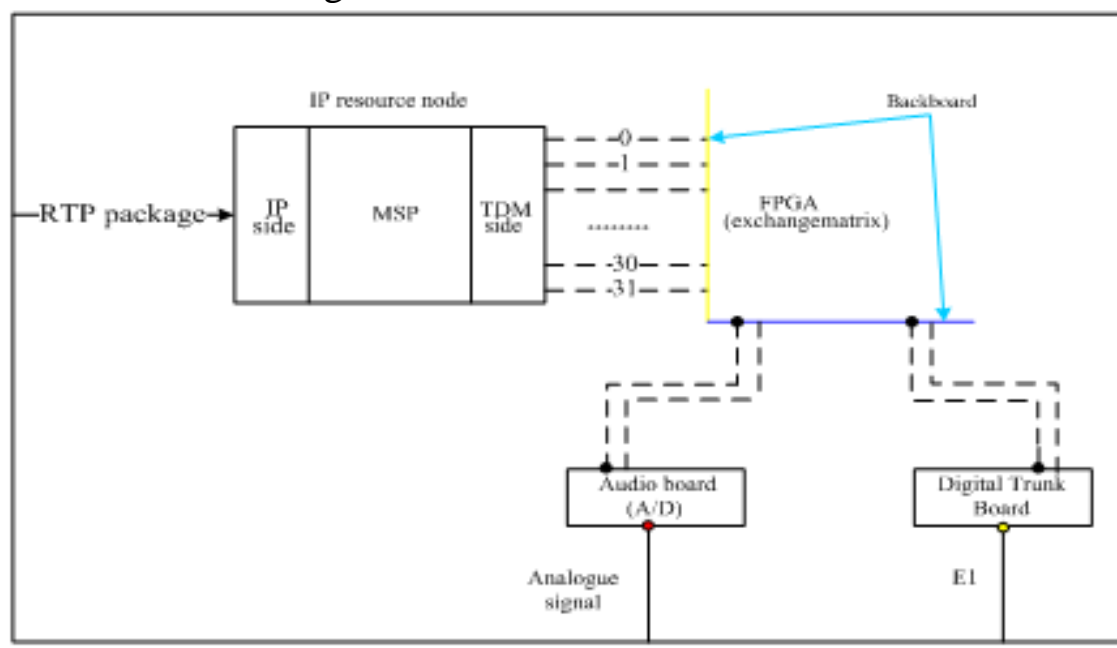

Fig. 7 Media Exchange

\section{Conclusion}

All in all, depending on the interface design, signaling exchange design and media exchange design, the multi-system convergence technique, based on the core exchange system -- digital dispatching system and the audio convergence device, improves the interfaces on the equipment involved in the audio convergence system when all voice commanding communication systems are stably operated. By such technical improvement, an effect "one-size-fits-all" is realized and the operation done on the terminal equipment by commander is greatly reduced. In this way, 
commander is given more time to think about the decision-making and the commander is able to know about the actual situations at the terminals; moreover, commander can give orders directly to the terminals. by the convergence technique, tasks can be distributed more flexibly and the authenticity of simulation can be greatly enhanced.

\section{Reference}

[1] Li Wei, Research and Design of Intercommunication Platform for Electronic Authentication Service at Yangtze River Delta, academic dissertation of Zhejiang University of Technology, 2014

[2] He Gaobo, Li Jun Automation of Processing Urban 3D Grid Data Shanxi-Shanxi-Hebei Coal Society Geologic Survey Seminar, 2006-09-1

[3] Zhang Shuqiang Application of Intelligent Voice Technology in Converged Communication, Academic Dissertation of Southeast University. 2012

[4] Zhang Chengwen, Sun Pengfei, Tan Xuezhi Multi-layer Interconnection of Trunk System, Electronic Device, Period 2 in 2015

[5] Lu Hongtao, Chen Yue, Qian Shaobo Discussion on Collaborative Guarantee for Multi-standard Digital Trunk System under Emergency, Mobile Communication, Period 13 in 2015

[6] Zhang Xiaofei Research on Audio and Video System used by Android Platforms, Player Development, Academic dissertation of Xidian University. 2012

[7] Qin Ning Research and Implementation of IP Internet Voice Transmission, Academic Dissertation of Beijing University of Posts and Telecommunications. 2005 